\title{
Neue Impulse für das FMH-Forum Notfall
}

\section{Claudia Schmidlin}

Projektassistentin, Abteilung Dienstleistungen \& Berufsentwicklung sowie Public Health, FMH

Das FMH-Forum Notfall ist 1995 auf Initiative einiger am Rettungswesen interessierter Fachgesellschaften unter dem Dach der FMH entstanden und hiess ursprünglich Plattform Schweizerisches Rettungswesen. Es engagiert sich für eine interdisziplinäre und integrierte Notfallmedizin jenseits von Partikularinteressen und regionalen Beschränkungen. Seit 2020 steht dabei die Neuorganisation der ärztlichen Notfallversorgung im Fokus, wie im neuen Leitbild festgehalten wurdeund seit Mai 2021 steht es unter der Leitung von Stefan Müller.

\begin{abstract}
Stefan Müller, an der Forumssitzung vom 18. Mai 2021 wurden Sie zum neuen Präsidenten gewählt. Wie kam es dazu?

Ich habe seit rund zehn Jahren als Vertreter der SGNOR für die Präklinik Einsitz im FMH-Forum Notfall. Der damalige Präsident Wolfgang Ummenhofer hatte mich zu jener Zeit angefragt. Als er Ende letzten Jahres bekanntgab, das Präsidium abgeben zu wollen, habe ich mich - das muss ich zugeben - nicht vorgedrängt. Doch ich war und bin überzeugt, dass das Forum eine wichtige Austauschplattform für Vertreterinnen und Vertreter verschiedenster Fachdisziplinen mit mehr oder weniger Berührungspunkten zum Thema Notfall ist. Die behandelten Themen führen zwar nicht gleich zu Änderungen im Gesundheitswesen; nichtsdestotrotz sind sie meines Erachtens wichtig. Das Fortbestehen des Forums lag mir denn auch am Herzen.
\end{abstract}

Wolfgang Ummenhofer hat das Forum Notfall jahrelang geführt und massgeblich geprägt. In welchem Stil planen Sie das Forum fortzuführen?

Die Fussstapfen von Wolfgang Ummenhofer sind riesig er ist quasi einer der Väter der präklinischen Notfallmedizin in der Schweiz -, und sie zu füllen ist gar nicht möglich. Wichtig ist mir ein partizipativer Ansatz: Das

Das Forum soll keine "One-Man-Show» sein, sondern verlangt das Engagement aller Mitglieder.

Forum soll keine «One-Man-Show» sein, sondern verlangt das Engagement aller Mitglieder. Ich sehe mich eher als Organisator oder Koordinator, der dem Gan-

\section{Zur Person}

Stefan Müller ist in Bern geboren und aufgewachsen. Er hat an der Uni Bern studiert und doktoriert und danach während fast 3 Jahren als Postdoc an der Harvard Medical School in Boston geforscht. Das Curriculum führte ihn von der Unfallchirurgie über die Intensivmedizin zur Anästhesiologie, in der er den Facharzttitel erwarb. Seit 16 Jahren

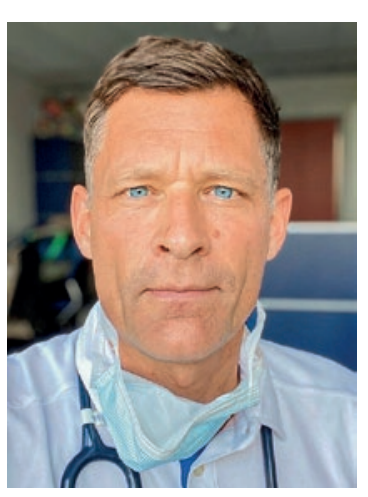
arbeitet er bei Schutz \& Ret-

tung Zürich in einem 50\%-Pensum, seit 2013 als deren Chefarzt; daneben arbeitet er zu 50\% als Leitender Arzt am Institut für Anästhesie und Intensivmedizin am Stadtspital Zürich. Über die Jahre absolvierte er den Fähigkeitsausweis "Notarzt SGNOR», das Zertifikat "Leitender Notarzt» sowie ein CAS zu "Systemisch Integratives Management im Gesundheitswesen». Er ist Instruktor für diverse Notfallkurse $\left(\mathrm{ACLS}^{\circledast}, \mathrm{ATLS}^{\oplus}, \mathrm{PHTLS}^{\circledast}, \mathrm{SFG}-\mathrm{P}\right)$, hat Einsitz in diversen standespolitischen Gremien und Gesellschaften unter anderem als Vorstandsmitglied der SGNOR sowie als Präsident von deren Bildungskommission. Daneben ist er seit vielen Jahren ein Leitender Notarzt am WEF. Er ist Oberst im Stab derTerritorialdivision 4.

zen etwas Struktur gibt. Da stimmt mich der Blick auf die Mitgliederliste zuversichtlich, viele kenne ich persönlich. Aktuell sind wir daran, das Feld etwas zu öffnen und Vertretende von Organisationen mit jüngeren Mitgliedern zur Teilnahme einzuladen. Davon erhoffen wir uns weitere spannende Inputs. 


\section{Welche Themen beschäftigen das FMH-Forum Notfall aktuell?}

In den vergangenen Monaten haben wir uns unter anderem mit dem Stroke-Management auseinandergesetzt sowie dem SWISSRECA, einem webbasierten, nationalen Herzstillstand-Langzeitregister, das erstmals prähospitale mit klinischen Daten kombinieren soll. Beiträge kommen dabei jeweils von unseren Mitgliedern, aber auch geladenen Gästen, die dem Forum ihre Projekte vorstellen und die Türe für weitere Gespräche öffnen. In diesem Zusammenhang ist für kommende Sitzungen auch die End-of-Life-Thematik traktandiert. Als Notärztin oder Notarzt sieht man immer wieder ältere Patientinnen und Patienten, die keine oder nur eine unvollständige Patientenverfügung haben. Sogar in Alters- und Pflegeheimen fehlen solche Informationen gelegentlich. Dabei wäre es doch eigentlich äusserst wichtig und wünschenswert, den Letzten Willen des Patienten oder der Patientin zu kennen und zu erfüllen. Das ist ein Thema, welches zahlreiche Fachrichtungen involviert, und entsprechend birgt das FMH-Forum Notfall die Chance auf gewinnbringende Diskussionen und Erkenntnisse.

\section{Welche Herausforderungen und Perspektiven sehen Sie für das Forum Notfall?}

Bei der «Rettungskette» müssen wir ganz am Anfang ansetzen, bei den Laien. In anderen Ländern ist es Standard, dass an Schulen Reanimationsausbildungen durchgeführt werden. Dadurch, dass die Kinder zu Hause erzählen, was sie erlebt haben, entsteht ein effektiver und sehr wertvoller Skalierungseffekt. Davon wird in der Schweiz noch nicht optimal profitiert.

In anderen Ländern ist es Standard, dass an Schulen Reanimationsausbildungen durchgeführt werden.

Ausserdem sind die Schulen ein fast alternativloser Multiplikator, wenn wir wirklich langfristig und nachhaltig alle Schweizerinnen und Schweizer erreichen wollen. Das wird weltweit so gesehen, und wir wollen uns dafür einsetzen, dass sich auch in der Schweiz bald etwas positiv verändert. Weitere "Player» der Rettungskette, wie beispielsweise die "First Responder» sind schon besser etabliert, könnten aber auch im nationalen Kontext von den Pilot-Initiativen profitieren. Das Niveau des professionellen Rettungsdienstes und des Notfallmanagements im klinischen Bereich ist in der Schweiz sehr hoch. Doch auch gerade diese Strukturen haben einen grossen interdisziplinären Background und profitieren sicher, wenn wir uns als Forum in Zukunft noch stärker mit ihnen auseinandersetzen und Vorschläge zur besseren Vernetzung und Optimierung erarbeiten.

\section{Worauffreuen Sie sich in der Arbeit im Forum?}

Der Austausch und die Zusammenarbeit mit engagierten Kolleginnen und Kollegen gefallen mir sehr; ich hoffe, später zu sehen, dass unser Wirken Früchte trägt

\section{Der Austausch und die Zusammenarbeit} mit engagierten Kolleginnen und Kollegen gefallen mir sehr.

und wir etwas initiieren können. Im föderalistischen Gesundheitssystem der Schweiz ist es nicht einfach, etwas zu bewegen. Ich würde mich sehr freuen, wenn wir es schaffen könnten, dank dieser interdisziplinären Zusammenarbeit Spuren zu hinterlassen. Konkret soll es auch ein Anliegen des Forums und der FMH sein, die interdisziplinäre Notfallmedizin mit einer einheitlichen und zeitgemässen Ausbildungsstruktur in die Curricula der schweizerischen Medizinischen Fakultäten zu integrieren. Hier hat die FMH bereits seit vielen Jahren den Anfang gemacht, indem sie das Mandat als Akkreditierungsinstanz für den Dienstarztkurs der SGNOR und das sogenannte Longitudinale Curriculum Notfallmedizin im Medizinstudium übernommen hat. Hierbei sollen prähospitale Versorgungsziele und klinisches Notfallmanagement einheitlich und praxisnah für alle Medizinstudierenden vermittelt werden.

Die Notfallmedizin ist ein ideales Feld, um im Interesse der Patienten und Patientinnen, die am meisten auf eine schnelle und kompetente Hilfe angewiesen sind, von der Expertise der beteiligten Fachdisziplinen und der Optimierung der involvierten Versorgungsstrukturen und Schnittstellen profitieren zu können. Hier will das Forum Notfall sowohl dem ärztlichen Grundversorgenden als auch dem medizinischen Nachwuchs den Weg erleichtern.

Das Wichtigste zum FMH-Forum Notfall erfahren Sie im 202 erschienenen Leitbild der FMH unter saez.ch/article/doi/saez. 2021.19573 